Annals of Pure and Applied Mathematics

Vol.19, No.1, 2019, 101-110

ISSN: 2279-087X (P), 2279-0888(online)

Published on 7 March 2019

Annals of

www.researchmathsci.org

DOI: http://dx.doi.org/10.22457/apam.600v19n1a14

Pure and Applied

Mathematics

\title{
Generalized Birkhoff Center of Almost Distributive Lattices
}

\author{
Berhanu Assaye Alaba ${ }^{1}$, Mihret Alamneh Taye ${ }^{2}$ and Gezahagne Mulat Addis ${ }^{3}$ \\ Department of Mathematics, Bahir Dar University \\ Bahir Dar, Ethiopia. \\ 1email: berhanu assaye@yahoo.com \\ 2email: mihretmahlet@yahoo.com; ${ }^{3}$ buttu412@yahoo.com
}

Received 10 February 2019; accepted 6 March 2019

\begin{abstract}
In this paper we define sectional Birkhoff center for an Almost Distributive Lattice not necessarily with maximal elements; as a Birkhoff center of its principal ideals. Moreover, we extend this result and define the generalized Birkhoff center of an ADL not necessarily with maximal elements. We give a necessary and sufficient condition for an ADL to be relatively complemented in terms of its sectional Birkhoff centers. Also we define and characterize sectionaly complemented ideals and sectional factor congruences using sectional Birkhoff centers
\end{abstract}

Keywords: Birkhoff center of ADLs; Sectional Birkhoff center of ADLs; generalized Birkhoff center of ADLs

AMS Mathematics Subject Classification (2010): 06D99

\section{Introduction}

Several algebraic structures have been studied by different scholars as a generalization for distributive lattices for instance near lattices (see [9]), skew lattices (see [10]), 0distributive and 1-distributive lattices (see $[2,11,16$ ), 0-distributive near lattices (see [8, 5, 6]), 0-distributive and 1-distributive semilattices (see [1, 7]), almost distributive lattices (see 12,15]), etc.

The concept of almost distributive lattices (or shortly an ADL) was first introduced by Swamy and Rao [13] in 1980. An ADL is an algebra with two binary operations $\wedge$ and $\vee$ which satisfies most of the properties of a distributive lattice with smallest element 0 except the commutativity of the binary operations $\Lambda$ and $V$.

It is well known that the Birkhoff centre of a bounded partially ordered set $P$ is a Boolean algebra in which the operations are l.u.b. and g.l.b. in P. In [14], Swamy, Rao, Ravi Kumar and Pragathi have extended the above concept for a general partially ordered set $P$ and proved that $B(P)$ is a relatively complemented distributive lattice in which the operations are 1.u.b. and g.l.b, in $P$ (provided $B(P)$ is non-empty). In [15], U.M.Swamy, and Murthy, studied Boolean center of algebras in a more general context; in universal algebra. Later in 2009 Swamy and Ramesh [12] have been introduced the concept of Birkhoffcentre $B(L)$ of an Almost Distributive Lattice $L$ with maximal elements and they have proved that $B(L)$ is a relatively complemented almost distributive lattice. Also they 


\section{Yu-dong Sun and Yi-min Shi}

have proved that an ADL $L$ with maximal elements is relatively complemented if and only if $B(L)=L$. Moreover they have obtained one-to-one correspondences between the Birkhoff centre $B(L)$ of $L$ and the set of complemented ideals of $L$ and between the Birkhoff center of $L$ and the set of factor-congruences on $L$. The question in this case is that what can we say about the Birkhoff center of an ADL with no maximal elements? In this paper we answer this question in a positive way by defining sectional Birkhoff centers of an Almost Distributive Lattice $L$ (not necessarily with maximal elements). We also prove certain results analogous to those results in [12]. Mainly we give a set of equivalent conditions conditions for an ADL (not necessarily with maximal elements) to be relatively complemented in terms of its sectional Birkhoff centers. In addition we define sectionaly complemented ideals (respectively sectional factor congruences) of ADLs and we characterize them using sectional Birkhoff centers. Finally we extend the result and define the generalized Birkhoff center of an ADL $L$ not necessarily with maximal elements and we give some characterizations.

\section{Preliminaries}

In this section, we recall some necessary definitions and results on ADLs which will be used in the paper. We refer to [3,4] for those standard concepts on distributive lattices and general universal algebras.

Definition 2.1. [4] An algebra $(L, \vee, \wedge, 0)$ of type $(2,2,0)$ is called an Almost Distributive Lattice, abbreviated as ADL, if it satisfies the following axioms;
(1) $a \vee 0=a$
(2) $0 \wedge a=0$
(3) $(a \vee b) \wedge c=(a \wedge c) \vee(b \wedge c)$
(4) $a \wedge(b \vee c)=(a \wedge b) \vee(a \wedge c)$
(5) $a \vee(b \wedge c)=(a \vee b) \wedge(a \vee c)$
(6) $(a \vee b) \wedge b=b$

Example 2.2. [4] Let $X$ be a nonempty set. Fix $x_{0} \in X$. For any $x, y \in X$ define $\wedge$ and $\vee$ as follows

$$
x \wedge y=\left\{\begin{array}{ll}
x_{0} & \text { if } x=x_{0} \\
y & \text { if } x \neq x_{0}
\end{array} \text { and } x \vee y= \begin{cases}y & \text { if } x=x_{0} \\
x & \text { if } x \neq x_{0}\end{cases}\right.
$$

Then $\left(X, \vee, \wedge, x_{0}\right)$ is an $A D L$ with $x_{0}$ as its 0 .

From now onwards we write simply $\mathrm{L}$ for an $\operatorname{ADL}(\mathrm{L}, \mathrm{V}, \wedge, 0)$ unless and otherwise mentioned.

Lemma 2.3. [4] For any $a \in L$, we have
(1) $a \wedge 0=0$
(2) $0 \vee a=a$
(3) $a \wedge a=a$
(4) $a \vee a=a$

Lemma 2.4. [4] For any $a, b \in L$, we have
(1) $(a \wedge b) \vee b=b$
(2) $a \vee(a \wedge b)=a=a \wedge(a \vee b)$
(3) $a \vee(b \wedge a)=a=(a \vee b) \wedge a$ 


\section{Generalized Birkhoff Center of ADLs}

Corollary 2.5. [4] For any $a, b \in L$, we have

(1) $a \vee b=$ aif and only if $a \wedge b=b$

(2) $a \vee b=b$ if and only if $a \wedge b=a$

In view of the above corollary, we give the following

Definition 2.6. [4] For any $a, b \in L$, we say that $a$ is less than or equals to $b$ and we write $a \leq b$ if $a \wedge b=a$ or equivalently $a \vee b=b$.

Theorem 2.7. [4] For any $a, b \in L$, the following are equivalent

(1) $(a \wedge b) \vee a=a$

(2) $a \wedge(b \vee a)=a$

(3) $(b \wedge a) \vee b=b$

(4) $b \wedge(a \vee b)=b$

(5) $a \wedge b=b \wedge a$

(6) $a \vee b=b \vee a$

(7) The supremum of $a$ and $b$ exists in $L$ and equals $a \vee b$

(8) There exists $x \in L$ such that $a \leq x$ and $b \leq x$

(9) The infimum of $a$ and $b$ exists in $L$ and equals $a \wedge b$

Theorem 2.8. [4] For any $a, b, c \in L$, we have

(1) $(a \vee b) \wedge c=(b \vee a) \wedge c$

(2) $\wedge$ is associative in $L$ and

(3) $a \wedge b \wedge c=b \wedge a \wedge c$

From the above theorem it follows that for any $x \in L$, the set $\{a \wedge x: a \in L\}$ forms a bounded distributive lattice and in particular we have $((a \wedge b) \vee c) \wedge x=((a \vee c) \wedge$ $(b \vee c)) \wedge x$ for all $a, b, c, x \in L$.

An element $m \in L$ is said to be maximal in $L$ if $m \leq x$ implies $m=x$. It can be easily observed that $m$ is maximal if and only if $m \wedge x=x$ for all $x \in L$.

Definition 2.9. [4] A nonempty set I in an $A D L L$ is called an ideal of $L$, if:

(1) $a, b \in I \Longrightarrow a \vee b \in I$

(2) $a \in I$ and $x \in L \Rightarrow a \wedge x \in I$

If $I$ is an ideal of $L$ and $a \in I$, then $x \wedge a \in I$ for all $x \in L$; For, $x \wedge a=x \wedge a \wedge a=$ $a \wedge x \wedge a \in I$.

Definition 2.10. [4] Let $L$ be an ADL. For any $a \in L$, the set $(a]=\{a \wedge x: x \in L\}$ is called a principal ideal of $L$ generated by $a$ and the set $[a)=\{x \vee a: x \in L\}$ is called the principal filter of $L$ generated by $a$.

Note that the principal ideal ( $a$ ] of $L$ is also a subADL of $L$ with maximal elements (since $a$ itself is maximal) and an element $b$ is maximal in ( $a$ ] if and only if $a \wedge b=b$ and $b \wedge a=a$ or equivalently if $(a]=(b]$. 


\section{Yu-dong Sun and Yi-min Shi}

\section{Sectional Birkhoff center}

In this section we define sectional Birkhoff center $B_{a}(L)$ of an Almost Distributive Lattice $L$ (not necessarily with maximal elements) determined by each elements $a \in L$.

Definition 3.1. Given an $A D L L$ and $a \in L$, define

$B_{a}(L)=\{x \in L: \exists y \in(a]$ such that $x \wedge y=0$ and $x \vee y$ is maximal in $(a]\}$

For each $a \in L$, we call $B_{a}(L)$ the sectional Birkhoff center of $L$ determined by $a$. In other words, this $B_{a}(L)$ is the Birkhoff center of a subADL ( $a$ ] of $L$.

For any $a \in L$ we have $a \wedge 0=0=0 \wedge$ and $a \vee 0=a=0 \vee a$. Thus $0, a \in B_{a}(L)$ and hence $B_{a}(L)$ is nonempty for all $a \in L$.

Lemma 3.2. If $m$ is a maximal element in $L$, then $B_{m}(L)=B(L)$; the Birkhoff center of $L$ introduced in [3].

Lemma 3.3. For any $a, b \in L$ we have the following

(1) $B_{a}(L) \subseteq(a]$

(2) $B_{a}(L)=\{0\}$ if and only ifa $=0$.

\section{Proof:}

(3) $B_{a}(L)=B_{b}(L)$ if and only if $(a]=(b]$

(1) $x \in B_{a}(L) \Rightarrow$ there exists $y \in(a]$ such that $x \wedge y=0$ and $x \vee y$ is maximal in (a]; that is, $(x \vee y) \wedge a=a$ and $a \wedge(x \vee y)=(x \vee y)$. Now from $(x \vee y) \wedge$ $a=a$ we have $a \wedge x=((x \vee y) \wedge a) \wedge x=(a \wedge(x \vee y)) \wedge x=(x \vee y) \wedge x=$ $x$. Therefore $x \in(a]$.

(2) Since $a \in B_{a}(L), B_{a}(L)=\{0\} \Rightarrow a=0$. On the otherhand if $a=0$ since $B_{a}(L) \subseteq(a]=\{0\}$, then we get that $B_{a}(L)=\{0\}$.

(3) Let $B_{a}(L)=B_{b}(L)$, then we have $a \in B_{a}(L)=B_{b}(L) \subseteq(b] \Rightarrow a \in(b]$ and $b \in B_{b}(L)=B_{a}(L) \subseteq(a] \Longrightarrow b \in(a]$. Therefore $(a]=(b]$. The converse is clear.

Theorem 3.4. Let $L$ be an $A D L$ and $a \in L$. Then $x \in B_{a}(L)$ if and only if there exists two ADLs $L_{1}$ and $L_{2}$ with maximal elements $m_{1}$ and $m_{2}$ respectively and an isomorphism $f:(a] \rightarrow L_{1} \times L_{2}$ such that $f(x)=\left(m_{1}, 0\right)$.

Proof: Suppose that $x \in B_{a}(L)$. Then there exists $y \in(a]$ such that $x \wedge y=0$ and $x \vee y$ is maximal in (a]; that is, $(x \vee y) \wedge a=a$ and $a \wedge(x \vee y)=(x \vee y)$. Put $L_{1}=(x]=$ $\{x \wedge z: z \in L\}$ and $L_{2}=(y]=\{y \wedge z: z \in L\}$. Then both $L_{1}$ and $L_{2}$ are ADLs with maximal elements $x$ and $y$ respectively. Now define $f:(a] \rightarrow L_{1} \times L_{2}$ by $f(a \wedge z)=$ $(x \wedge z, y \wedge z)$ for all $z \in L$. Then $f(x)=f(a \wedge x)=(x \wedge x, y \wedge x)=(x, 0)$. We show that $f$ is an isomorphism. Clearly $f$ is a homomorphism and To prove $f$ is one-one, let $z_{1}, z_{2} \in L$ such that $f\left(a \wedge z_{1}\right)=f\left(a \wedge z_{2}\right)$

$$
\begin{aligned}
& \Rightarrow\left(x \wedge z_{1}, y \wedge z_{1}\right)=\left(x \wedge z_{2}, y \wedge z_{2}\right) \\
& \Rightarrow x \wedge z_{1}=x \wedge z_{2} \text { and } y \wedge z_{1}=y \wedge z_{2}
\end{aligned}
$$

Now consider the following;

$$
a \wedge z_{1}=((x \vee y) \wedge a) \wedge z_{1}
$$

$$
\begin{aligned}
& =(a \wedge(x \vee y)) \wedge z_{1} \\
& =(x \vee y) \wedge z_{1}
\end{aligned}
$$




\section{Generalized Birkhoff Center of ADLs}

$$
\begin{aligned}
& =\left(x \wedge z_{1}\right) \vee\left(y \wedge z_{1}\right) \\
& =\left(x \wedge z_{2}\right) \vee\left(y \wedge z_{2}\right) \\
& =(x \vee y) \wedge z_{2} \\
& =(a \wedge(x \vee y)) \wedge z_{2} \\
& =((x \vee y) \wedge a) \wedge z_{2} \\
& =a \wedge z_{2}
\end{aligned}
$$

This says that $f\left(a \wedge z_{1}\right)=f\left(a \wedge z_{2}\right) \Rightarrow a \wedge z_{1}=a \wedge z_{2}$. Hence $f$ is one-one. It remains to show that $f$ is onto. Let $u \in L_{1} \times L_{2}$, then $u=\left(u_{1}, u_{2}\right)$ such that $u_{1} \in L_{1}=(x]$ and $u_{2} \in L_{2}=(y]$. Then $u_{1}=x \wedge z_{1}$ for some $z_{1} \in L$ and $u_{2}=y \wedge z_{2}$ for some $z_{2} \in L$. So that $u=\left(x \wedge z_{1}, y \wedge z_{2}\right)$ for some $z_{1}$ and $z_{2} \in L$. Put $z=\left(x \wedge z_{1}\right) \vee\left(y \wedge z_{2}\right)$. Then consider;

$$
\begin{aligned}
x \wedge z & =x \wedge\left(\left(x \wedge z_{1}\right) \vee\left(y \wedge z_{2}\right)\right) \\
& =\left(x \wedge\left(x \wedge z_{1}\right)\right) \vee\left(x \wedge\left(y \wedge z_{2}\right)\right) \\
& =\left(x \wedge z_{1}\right) \vee\left(x \wedge y \wedge z_{2}\right) \\
& =\left(x \wedge z_{1}\right) \vee 0 \\
& =x \wedge z_{1}
\end{aligned}
$$

Similarly;

$$
\begin{aligned}
y \wedge z & =y \wedge\left(\left(x \wedge z_{1}\right) \vee\left(y \wedge z_{2}\right)\right) \\
& =\left(y \wedge\left(x \wedge z_{1}\right)\right) \vee\left(y \wedge\left(y \wedge z_{2}\right)\right) \\
& =\left(y \wedge x \wedge z_{1}\right) \vee\left(y \wedge z_{2}\right) \\
& =0 \vee\left(y \wedge z_{2}\right) \\
& =y \wedge z_{2}
\end{aligned}
$$

Therefore $u=\left(x \wedge z_{1}, y \wedge z_{2}\right)=(x \wedge z, y \wedge z)$ where $z=\left(x \wedge z_{1}\right) \vee\left(y \wedge z_{2}\right) \in L$. Thus $u=f(a \wedge z)$ for some $a \wedge z \in(a]$ and hence $f$ is onto. Hence $f$ is an isomorphism. Conversely suppose that there exists two ADLs $L_{1}$ and $L_{2}$ with maximal elements and an isomorphism $f:(a] \rightarrow L_{1} \times L_{2}$ such that $f(x)=\left(m_{1}, 0\right)$ where $m_{1}$ is a maximal element in $L_{1}$. Choose a maximal element $m_{2}$ in $L_{2}$. Since $f$ is an isomorphism there exists $y \in(a]$ such that $f(y)=\left(0, m_{2}\right)$. Consider; $f(x \wedge y)=f(x) \wedge f(y)=$ $\left(m_{1}, 0\right) \wedge\left(0, m_{2}\right)=\left(m_{1} \wedge 0,0 \wedge m_{2}\right)=(0,0)$ (a zero element in $\left.L_{1} \times L_{2}\right)$. Since $f$ is an isomorphism it follows that $x \wedge y=0$. Also $f(x \vee y)=f(x) \vee f(y)=\left(m_{1}, 0\right) \vee$ $\left(0, m_{2}\right)=\left(m_{1} \vee 0,0 \vee m_{2}\right)=\left(m_{1}, m_{2}\right)$ which is maximal in $L_{1} \times L_{2}$. Since $f$ is an isomorphism we get $x \vee y$ to be maximal in (a] which implies that $y$ is the complement of $x$ in $(a]$. So that $x \in B_{a}(L)$. Hence the result.

Theorem 3.4. For any $a$ in an $A D L L, B_{a}(L)$ is relatively complemented $A D L$.

Proof: Clearly we have $0 \in B_{a}(L)$ and hence $B_{a}(L) \neq \emptyset$.

Let $x_{1}, x_{2} \in B_{a}(L)$. Then there exists $y_{1}$ and $y_{2}$ in $(a]$ such that $x_{1} \wedge y_{1}=0, x_{1} \vee y_{1}=$ $m_{1}$ and $x_{2} \wedge y_{2}=0, x_{2} \vee y_{2}=m_{2}$ where $m_{1}$ and $m_{2}$ are maximal elements in ( $a$ ]; that is, $\left(m_{1}\right]=(a]=\left(m_{2}\right]$.

Now consider;

$$
\begin{aligned}
& \left(x_{1} \wedge x_{2}\right) \wedge\left(y_{1} \vee y_{2}\right)=\left[\left(x_{1} \wedge x_{2}\right) \wedge y_{1}\right] \vee\left[\left(x_{1} \wedge x_{2}\right) \wedge y_{2}\right] \\
& \quad=\left(x_{1} \wedge x_{2} \wedge y_{1}\right) \vee\left(x_{1} \wedge x_{2} \wedge y_{2}\right) \\
& \quad=\left(x_{2} \wedge x_{1} \wedge y_{1}\right) \vee\left(x_{1} \wedge x_{2} \wedge y_{2}\right) \\
& \quad=\left(x_{2} \wedge 0\right) \vee\left(x_{1} \wedge 0\right) \\
& \quad=0
\end{aligned}
$$


Yu-dong Sun and Yi-min Shi

Also for any $t \in(a]$ we have;

$$
\begin{aligned}
{\left[\left(x_{1} \wedge x_{2}\right) \vee\right.} & \left.\left(y_{1} \vee y_{2}\right)\right] \wedge t=\left[\left(x_{1} \vee y_{1} \vee y_{2}\right) \wedge\left(x_{2} \vee y_{1} \vee y_{2}\right)\right] \wedge t \\
& =\left[\left(m_{1} \vee y_{2}\right) \wedge\left(m_{2} \vee y_{1}\right)\right] \wedge t \\
& =\left(m_{1} \wedge m_{2}\right) \wedge t \\
& =m_{2} \wedge t \\
& =t
\end{aligned}
$$

This says that $\left(x_{1} \wedge x_{2}\right) \vee\left(y_{1} \vee y_{2}\right)$ is maximal in $(a]$. Therefore $y_{1} \vee y_{2}$ is the complement of $x_{1} \wedge x_{2}$ in $(a]$ and hence $x_{1} \wedge x_{2} \in B_{a}(L)$. Similarly we get that $y_{1} \wedge y_{2}$ is the complement of $x_{1} \vee x_{2}$ in $(a]$ and hence $x_{1} \vee x_{2} \in B_{a}(L)$. Thus $B_{a}(L)$ is a sub ADL of $L$.

Next we prove that $B_{a}(L)$ is relatively complemented. Let $x_{1}, x_{2} \in B_{a}(L)$. Then there exists $y_{1}$ and $y_{2}$ in $(a]$ such that $x_{1} \wedge y_{1}=0, x_{1} \vee y_{1}=m_{1}$ and $x_{2} \wedge y_{2}=0$, $x_{2} \vee y_{2}=m_{2}$ where $m_{1}$ and $m_{2}$ are maximal elements in $(a]$; that is, $\left(m_{1}\right]=(a]=$ $\left(m_{2}\right]$. Put $u=y_{1} \wedge x_{2}$ and $v=x_{1} \vee y_{2}$. Since $x_{1}, x_{2}, y_{1}$ and $y_{2} \in(a]$ then both $u$ and $v$ are in $(a]$.

Now consider;

$$
\begin{aligned}
& u \wedge v=\left(y_{1} \wedge x_{2}\right) \wedge\left(x_{1} \vee y_{2}\right) \\
& =\left(y_{1} \wedge x_{2} \wedge x_{1}\right) \vee\left(y_{1} \wedge x_{2} \wedge y_{2}\right) \\
& =0
\end{aligned}
$$

Also for any $t \in(a]$ we have;

$$
\begin{aligned}
& \quad(u \vee v) \wedge t=\left[\left(y_{1} \wedge x_{2}\right) \vee\left(x_{1} \vee y_{2}\right)\right] \wedge t \\
& =\left[\left(y_{1} \vee x_{1} \vee y_{2}\right) \wedge\left(x_{2} \vee x_{1} \vee y_{2}\right)\right] \wedge t \\
& =\left[\left(m_{1} \vee y_{2}\right) \wedge\left(m_{2} \vee x_{1}\right)\right] \wedge t \\
& =\left(m_{1} \wedge m_{2}\right) \wedge t \\
& =m_{2} \wedge t \\
& =t
\end{aligned}
$$

This says that $u \vee v$ is maximal in (a]. Therefore $v$ is a complement of $u$ in (a] so that $u \in B_{a}(L)$. Also, $x_{1} \wedge u=x_{1} \wedge y_{1} \wedge x_{2}=0 \wedge x_{2}=0$ and $x_{1} \vee u=x_{1} \vee\left(y_{1} \wedge x_{2}\right)=$ $\left(x_{1} \vee y_{1}\right) \wedge\left(x_{1} \vee x_{2}\right)=m_{1} \wedge\left(x_{1} \vee x_{2}\right)=x_{1} \vee x_{2}$. Therefore $u$ is an element in $(a]$ such that $x_{1} \wedge u=0$ and $x_{1} \vee u=x_{1} \vee x_{2}$. That is $u=x_{1}{ }^{x_{2}}$ and hence $B_{a}(L)$ is relatively complemented.

Theorem 3.5. Let $L$ be an $A D L$. Then the following are equivalent

(1) Lis relatively complemented

(2) $(a]=B_{a}(L)$ for all $a \in L$

(3) For any $a$ and $b$ in $L$ we havea $\in B_{a v b}(L)$.

Proof: (1) $\Longrightarrow$ (2) suppose that $L$ is relatively complemented and let $a \in L$ be any element.

Claim: $(a]=B_{a}(L)$

Clearly we have $B_{a}(L) \subseteq(a]$. So that it remains to show that $(a] \subseteq B_{a}(L)$. Let $x \in(a]$ then, $a \wedge x=x$. Since $L$ is relatively complemented there exists an element $y=x^{a}$ such that $x \wedge y=0$ and $x \vee y=x \vee a$. That is, $x \wedge y=0$ and $x \vee y$ is maximal in $(a]$. This says that $y$ is a complement of $x$ in $(a]$ which implies that $\in B_{a}(L)$. Therefore $(a]=$ $B_{a}(L)$. 


\section{Generalized Birkhoff Center of ADLs}

$(2) \Rightarrow(3)$. Suppose that $(a]=B_{a}(L)$ for all $a \in L$. Let $a, b \in L$, then $a \vee b \in L$ and hence by our assumption $(a \vee b]=B_{a \vee b}(L)$. By one of the absorption laws, since $(a \vee b) \wedge$ $a=a$, the $a \in(a \vee b]=B_{a \vee b}(L)$. Thus $a \in B_{a \vee b}(L)$. Hence the result.

$(3) \Longrightarrow(1)$. Suppose that $a \in B_{a \vee b}(L)$ for all $a, b \in L$. Then there exists $x \in(a \vee b]$ such that $a \wedge x=0$ and $a \vee x=m$ where $m$ is a maximal element in $(a \vee b]$ (i.e. $m \wedge$ $(a \vee b)=a \vee b$ and $(a \vee b) \wedge m=m$.

Now $(a \vee x) \wedge(a \vee b)=m \wedge(a \vee b)$

$$
\begin{gathered}
\Rightarrow[a \wedge(a \vee b)] \vee[x \wedge(a \vee b)]=a \vee b \\
\Rightarrow a \vee[x \wedge(a \vee b)]=a \vee b
\end{gathered}
$$

Put $y=x \wedge(a \vee b)$ then $y \in(a \vee b]$ such that; $a \wedge y=a \wedge x \wedge(a \vee b)=0 \wedge(a \vee b)=$ 0 and $a \vee y=a \vee[x \wedge(a \vee b)]=a \vee b$. Therefore $y=a^{b}$. Hence $\mathrm{L}$ is relatively complemented.

Theorem 3.6. Let $L_{1}$ and $L_{2}$ be ADLs and $a_{1} \in L_{1}$ and $a_{2} \in L_{2}$. Then $B_{\left(a_{1}, a_{2}\right)}\left(L_{1} \times L_{2}\right)=B_{a_{1}}\left(L_{1}\right) \times B_{a_{2}}\left(L_{2}\right)$

Let $L$ be an ADL. The relation $\varphi=\{(x, y) \in L \times L: x \wedge y=y$ and $y \wedge x=x\}$ is a congruence relation on $L$ and is the smallest such that $L / \varphi$ is a distributive lattice. [6]

Theorem 3.7. Let $L$ be an $A D L$ and $a \in L$. Then $B_{\varphi(a)}(L / \varphi)$ is isomorphic to $B_{a}(L) /$

$$
\varphi \cap\left(B_{a}(L) \times B_{a}(L)\right)
$$

Proof: Define $f: B_{a}(L) \rightarrow B_{\varphi(a)}(L / \varphi)$ by $f(x)=\varphi(x)$ for all $x \in B_{a}(L)$. We first show that this $f$ is well defined. For; let $x \in B_{a}(L)$ then there exists $y \in(a]$ such that $x \wedge y=0$ and $x \vee y$ is maximal in $(a]$; that is, $(x \vee y) \wedge a=a$ and $a \wedge(x \vee y)=(x \vee y)$

$$
\begin{aligned}
& \Rightarrow x \wedge y=0 \text { and }(x \vee y, a) \in \varphi \\
& \Rightarrow \varphi(x \wedge y)=\varphi(0) \text { and } \varphi(x \vee y)=\varphi(a) \\
& \Rightarrow \varphi(x) \wedge \varphi(y)=\varphi(0) \text { and } \varphi(x) \vee \varphi(y)=\varphi(a) \\
& \Rightarrow \varphi(y) \text { is the complement of } \varphi(x) \text { in }(\varphi(a)] \\
& \Rightarrow \phi(x) \in B_{\varphi(a)}(L / \varphi) \\
& \Rightarrow f(x) \in B_{\varphi(a)}(L / \varphi)
\end{aligned}
$$

Therefore $f$ is well defined. It is also clear that $f$ is a homomorphism. Now we show that $f$ is an epimorphism. Let $\varphi(x) \in B_{\varphi(a)}(L / \varphi)$ for some $x \in L$, then there exists $\varphi(y) \in$ $(\varphi(a)]$ such that; $\varphi(x) \wedge \varphi(y)=\varphi(0)$ and $\varphi(x) \vee \varphi(y)=\varphi(a)$.

$\Rightarrow \varphi(x \wedge y)=\varphi(0)$ and $\varphi(x \vee y)=\varphi(a)$

$\Rightarrow(x \wedge y, 0) \in \varphi$ and $(x \vee y, a) \in \varphi$

$\Rightarrow x \wedge y=0$ and $(x \vee y) \wedge a=a$ and $a \wedge(x \vee y)=(x \vee y)$; that is, $x \vee y$ is

maximal in $(a]$

$$
\begin{aligned}
& \Rightarrow y \text { is the complement of } x \text { in }(a] \\
& \Rightarrow x \in B_{a}(L) \text { such that } f(x)=\varphi(x)
\end{aligned}
$$

Therefore $f$ is an epimorphism. Also consider its kernel;

$$
\begin{aligned}
& \operatorname{ker} f=\left\{(x, y) \in B_{a}(L) \times B_{a}(L): f(x)=f(y)\right\} \\
= & \left\{(x, y) \in B_{a}(L) \times B_{a}(L): \phi(x)=\varphi(y)\right\} \\
= & \left\{(x, y) \in B_{a}(L) \times B_{a}(L):(x, y) \in \varphi\right\}
\end{aligned}
$$




$$
=\varphi \cap\left(B_{a}(L) \times B_{a}(L)\right)^{\text {Yu-dong Sun and Yi-min Shi }}
$$

Therefore by the fundamental theorem of homomorphisms; ${ }_{a}(L) / \operatorname{ker} f$ is isomorphic to $B_{\varphi(a)}(L / \varphi)$; that is, $B_{a}(L) / \varphi \cap\left(B_{a}(L) \times B_{a}(L)\right)$ is isomorphic to $B_{\varphi(a)}(L / \varphi)$.

It is known that an ideal $I$ of an ADL $L$ is complemented there exists an ideal $J$ of $L$ sucth that $I \cap J=(0)$ and $I \vee J=L$. . In the following we define sectionaly complemented ideals:

Definition 3.8. An ideal I in an $A D L L$ is said to be sectionaly complemented relative to an ideal $J$ of $L$ if there exists an ideal $K$ of $L$ such that $I \cap K=(0)$ and $I \vee K=I \vee J$.

It observed in [4] that an ideal $I$ of an ADL $L$ is complemented if and only if $I=(a)$ for some $a \in L$. And later it has been proved in [3] that an An ideal $I$ of $L$ is complemented if and only if $I=(a)$ for some $a \in B(L)$. Analogously we have the following:

Theorem 3.9. Given ideals I and J of an ADL L. Iis sectionaly complemented relative to $J$ whenever $I=(a]$ and $J=(b]$ for some $a$ and $b$ in $L$ such that $a \in B_{a \vee b}(L)$

In the next theorem we characterize complemented ideals as sectionaly complemented ideals:

Theorem 3.10. $I$ is complemented if and only if $I$ is sectionaly complemented relative to each ideal $J$ of $L$.

Corollary 3.11. An ADL $L$ is relatively complemented if and only if the class PI $(L)$ of all principal ideals of $L$ is relatively complemented distributive lattice.

Given any filter $F$ of an ADL, define

$$
\phi_{F}:=\{(a, b) \in L \times L: x \wedge a=x \wedge \text { fforsomex } \in F\}
$$

Then $\phi_{F}$ is a congruence on $\mathrm{L}$. we write $\phi_{x}:=\{(a, b) \in L \times L: x \wedge a=x \wedge b\}$ for any $x \in L$. It is observed in [3] that for any $x \in L, \phi_{x}=L \times L$ if and only if $x=0$. A congruence $\theta$ on an ADL $L$ is said to be factor congruence if there exists a congruence $\phi$ on $L$ such that $\theta \cap \phi=\Delta$ and $\theta \circ \phi=L \times L$ [3]. In the following we extend this and we define sectional factor congruences.

Definition 3.12. Let $\theta_{1}$ and $\theta_{2}$ congruence relations on an ADL L. $\theta_{1}$ is said to be sectional factor congruence relative to $\theta_{2}$ if $\theta_{1}$ is a factor congruence in the interval $\left[\theta_{1} \cap \theta_{2}, L \times L\right]$. That is if there exists a congruence $\psi$ on $L$ such that $\theta_{1} \cap \psi=\theta_{1} \cap \theta_{2}$ and $\theta_{1} \circ \psi=L \times L$.

It is proved in [3] that a congruence $\theta$ on $L$ is a factor-congruence if and only if $\theta=\phi_{a}$ for some $a \in B(L)$. In the next theorem we extend this result to the case of sectional factor congruence relations: 


\section{Generalized Birkhoff Center of ADLs}

Theorem 3.13. Given congruence relations $\theta_{1}$ and $\theta_{2}$ on L. $\theta_{1}$ is a sectional factor congruence relative to $\theta_{2}$, whenever $\theta_{1}=\phi_{a}$ and $\theta_{2}=\phi_{b}$ for some $a$ and $b \in L$ such that $a \in B_{a \vee b}(L)$.

In the rest part of this section we define the generalized Birkhoff center $G B(L)$ of an almost distributive lattice $L$ not necessarily with maximal elements and we give some characterizing properties analogous to those results in [3].

Definition 3.14. Given an $A D L L$, define

$$
G B(L)=\left\{a \in L: a \in B_{a \vee b}(L) \text { for all } b \in L\right\}
$$

Not that, in the case $L$ has maximal elements, the generalized Birkhoff center $G B(L)$ of $L$ coincides with the Birkhoff center $B(L)$ of $L$. It is also easy to verify that the generalized Birkhoff center $G B(L)$ of $L$ is a relatively complemented ADL under the operations induced by those of operations in $L$. Moreover as a result of Theorem 3.5 it follows that $L$ is relatively complemented if and only if $G B(L)=L$.

Acknowledgements. We express our heartfelt thanks to the Chief-Editor for accepting our paper and suggestion for improvement of the paper.

\section{REFERENCES}

1. S.Akhter and A.S.A.Noor, 1-distributive join semilattice, J. Mech. Cont. \& Math. Sci., 7(2) (2013) 1067-1076.

2. P.Balasubramani and P.V.Venkatanarasimhan, Characterizations of the 0Distributive Lattices, Indian J. Pure Appl. Math., 32(3) (2001) 315-324.

3. G.Birkhoff, Lattice Theory, American Mathematical Society, Colloquium Publications, 1967

4. S.Buris, and A.Sankappanavar, A course in universal algebra, Springer-Verlag, New York, 1980

5. A.S.A.Noor, M.Ayub Ali and A.K.M.S.Islam, $\propto$-ideals in a distributive nearlattice, Journal of Physical Sciences, 16 (2012) 1-7.

6. Y.S.Pawar and M.A.Gandhi, 0-ideals in 0-distributive nearlattice, Annals of Pure and Applied Mathematics, 11 (2016) 57-65.

7. Y.S.Pawar and N.K.Thakare, 0-distributive semilattices, Canad. Math. Bull., 21(4) (1978) 469-475.

8. Md. Zaidur Rahman, Md. Bazlar Rahman and A.S.A.Noor, 0-distributive near lattice, Annals of Pure and Applied Mathematics, 2(2) (2012) 185-195.

9. D.Schweigert, Near lattices, Mathematica Slovaca 32(3) (1982) 313-317.

10. K.V.R Srinivas, Skew lattices, Proyecciones Journal of Mathematics, 30(1) (2011) 51-57

11. R.Sultana, Md. Ayub Ali and A.S.A.Noor, Some properties of 0-distributive and 1distributive lattices, Annals of Pure and Applied Mathematics, 1(2) (2012) 168- 175.

12. U.M.Swamy and S.Ramesh, Birkhoff center of an almost distributive lattice, Int. Jour. Algebra, 11 (2009) 539-546.

13. U.M.Swamy and G.C.Rao, Almost distributive lattices, Jour. Aust. Math. Soc. (Series A) 31 (1981) 77-91.

14. U.M.Swamy, G.C.Rao, R.V.G.Ravi Kumar and P.H.Pragathi, Birkhoff center of a poset, Southeast Asian Bulletin of Mathematics, 26 (2002) 509-516. 
Yu-dong Sun and Yi-min Shi

15. U.M.Swamy and G.S.N.Murthy, Boolean center of a Universal Algebra, Algebra Universalis, 13 (1981), 202-205.

16. J.C.Varlet, A generalization of the notion of pseudo-complementedness, Bull. Soc. Sci. Liege., 37 (1968) 149-158. 